\title{
Referenzwerte für den arteriellen Sauerstoffgehalt
}

Reference Values for Arterial Oxygen Content

Autoren

Institute
Ch. Warnke', T. Bollmann¹, T. Ittermann², S. Gläser ${ }^{1}$, B. Koch¹, Ch. Schäper ${ }^{1}$, K. Lau² , S. B. Felix ${ }^{1}$, R. Ewert ${ }^{1}$

${ }^{1}$ Klinik für Innere Medizin B, Universitätsmedizin Greifswald

${ }^{2}$ Institut für Community Medicine, Abteilung SHIP, Klinisch Epidemiologische Forschung, Universitätsmedizin Greifswald eingereicht $\quad 5.6 .2014$ akzeptiert nach Revision 6.8.2014

\section{Bibliografie}

DOI http://dx.doi.org/ 10.1055/s-0034-1378089 Online-Publikation: 8.10.2014 Pneumologie 2014; 68: 788-792 (c) Georg Thieme Verlag KG Stuttgart · New York ISSN 0934-8387

\section{Korrespondenzadresse} Dr. med. Christian Warnke Universitätsmedizin Greifswald Klinik für Innere Medizin B, Bereich Pneumologie, Infektiologie und Weaningzentrum 17475 Greifswald warnke@uni-greifswald.de

\section{Zusammenfassung \\ $\nabla$}

Hintergrund: Im klinischen Alltag wird zur Beurteilung des Sauerstoffbedarfs von Patienten regelmäßig die photoplethysmografisch gemessene Sauerstoffsättigung herangezogen. Ebenfalls breite Anwendung findet die Bestimmung des Sauerstoffpartialdrucks mittels Blutgasanalyse. Für das tatsächliche Sauerstoffangebot $\mathrm{DO}_{2}$ ist jedoch neben dem Herzzeitvolumen Q der arterielle Sauerstoffgehalt $\mathrm{c}_{\mathrm{a}} \mathrm{O}_{2}$ relevant. Für diesen Wert existieren bisher die 1984 von Zander und Mertzlufft sowie die 1990 von Siggaard-Andersen erstellten Normwerte. Ziel der vorliegenden Arbeit war, diese Normwerte im Rahmen einer populationsbasierten Studie nachzuvollziehen. Weiterhin sollte der Einfluss des Rauchens auf die Normwerte bestimmt werden.

Patienten und Methode: Für die Untersuchung wurden die Messwerte von 1018 Probanden der Study of Health in Pomerania (SHIP) ausgewertet. Der arterielle Sauerstoffgehalt wurde aus den Messwerten für Sauerstoffsättigung, Sauerstoffpartialdruck und Hämoglobinkonzentration aus arterialisiertem Kapillarblut entsprechend der modifizierten Hüfner-Formel berechnet. Mithilfe quantiler Regression und fraktionaler Polynome wurden alters- und geschlechtsspezifische Normwertgleichungen ermittelt.

Ergebnisse: Die unteren Normwerte für den arteriellen Sauerstoffgehalt sinken mit steigendem Alter. Aktuelles Rauchen hat keinen signifikanten Einfluss auf den unteren Normwert.

Schlussfolgerungen: Mit den vorgestellten Normwertgleichungen können individuelle Normwerte für erwachsene Patienten errechnet werden.

\section{Abstract \\ $\nabla$}

Background: In clinical practice, oxygen saturation as measured by photoplethysmography and arterial oxygen tension as determined by blood gas analysis are the parameters used frequently to estimate the oxygen status of a patient. Additionally, the cardiac output $\mathrm{CO}$ and the arterial oxygen content $\mathrm{CaO}_{2}$ are critical for the delivery of oxygen $\mathrm{DO}_{2}$ to organs and tissues. So far, $\mathrm{CaO}_{2}$ reference values published by Mertzlufft and Zander (1984) and Siggaard-Andersen (1990) are widely used. The aim of the present study was to reevaluate previously published results using the results of a population-based study. Furthermore, the impact of smoking on $\mathrm{CaO}_{2}$ will be assessed.

Patients and Methods: Data of 1018 volunteers from the Study of Health in Pomerania (SHIP) were analyzed. $\mathrm{CaO}_{2}$ was calculated from blood gas analysis of capillary blood obtained from a hyperemised ear lobe. Reference value equations controlled for sex, age and smoking were derived with quantile regression analysis and fractional polynomials.

Results: Lower limits of normal (LLN) decline with age. Current smoking has no significant influence on $\mathrm{LLN}$ for $\mathrm{CaO}_{2}$.

Conclusion: Sex, age and smoking-specific normal values can be calculated using the current equations. 


\section{Hintergrund und Zielstellung}

\section{$\nabla$}

Sauerstoff hat in der medizinischen Therapie einen großen Stellenwert [1]. Die Therapie wird dabei in der Regel über die photoplethysmografisch einfach zu messende Sauerstoffsättigung des Hämoglobins $\left(\mathrm{s}_{\mathrm{p}} \mathrm{O}_{2}\right)$ gesteuert. Zielparameter ist dabei meist eine Sättigung über 90\%. In der klinischen Routine findet zusätzlich die Blutgasanalyse aus arteriellem Blut oder arterialisiertem Kapillarblut, unter anderem zur Bestimmung des arteriellen Sauerstoffpartialdruckes $\left(\mathrm{p}_{\mathrm{a}} \mathrm{O}_{2}\right)$, breite Anwendung.

Das für die zelluläre Sauerstoffversorgung relevante Sauerstoffangebot $\left(\mathrm{DO}_{2}\right)$ errechnet sich als Produkt aus Herzminutenvolumen (CO) und arteriellem Sauerstoffgehalt $\left(\mathrm{c}_{\mathrm{a}} \mathrm{O}_{2}\right)$. Letzterer ist die Summe aus dem hämoglobingebundenen Sauerstoff, repräsentiert durch Hämoglobinkonzentration $(\mathrm{Hb})$ und Sauerstoffsättigung des Hämoglobins $\left(\mathrm{s}_{\mathrm{a}} \mathrm{O}_{2}\right)$ sowie einer sehr geringen Menge $(<2 \%)$ physikalisch gelösten Sauerstoffs, repräsentiert durch den $\mathrm{p}_{\mathrm{a}} \mathrm{O}_{2}$. In der klinischen Praxis wird allerdings dem Sauerstoffgehalt oft eine geringere Bedeutung als den Teilkomponenten beigemessen. In der Tat gilt vor allem der $\mathrm{p}_{\mathrm{a}} \mathrm{O}_{2}$-Wert oft als Grundlage der Therapiesteuerung und ist so auch zum Beispiel in der Leitlinie für die Langzeitsauerstofftherapie festgeschrieben [2], obwohl gerade bei gleichsinnigen Änderungen von $\mathrm{Hb}$ und $\mathrm{p}_{\mathrm{a}} \mathrm{O}_{2}$ der arterielle Sauerstoffgehalt stark schwanken kann.

Mertzlufft und Zander haben 1984 Normwerte für den mit der „O $\mathrm{O}_{2}$-Cuvette“ gemessenen arteriellen Sauerstoffgehalt, gemessen bei 455 Gesunden, publiziert [3]. Weitere Normwerte wurden von Siggaard-Andersen et al. 1990 veröffentlicht [4]. Diese Werte wurden aus Blutgasanalysen bei 35 gesunden Freiwilligen errechnet.

Die Menge des an Hämoglobin gebundenen Sauerstoffs ist das Produkt aus Hämoglobinkonzentration ( $\mathrm{Hb}$ ), Sauerstoffsättigung $\left(\mathrm{s}_{\mathrm{a}} \mathrm{O}_{2}\right)$ des Hämoglobins und einer Konstante $(\mathrm{k})$. Zuerst wurde $\mathrm{k}$ durch Hüfner mit 1,34 ml Sauerstoff pro Gramm Hämoglobin bestimmt [5]. Aktuell gültig ist der Wert für $\mathrm{k}$ von $1,39 \mathrm{ml} / \mathrm{g}$ [6]. Diesem liegt das massenspektrometrisch bestimmte Molekulargewicht des Hämoglobins zugrunde.

Somit ist die heute gültige Formel zur Berechnung des arteriellen Sauerstoffgehaltes $\left(\mathrm{c}_{\mathrm{a}} \mathrm{O}_{2}\right)$ :

$\mathrm{c}_{\mathrm{a}} \mathrm{O}_{2}[\mathrm{ml} / \mathrm{l}]=1,39[\mathrm{ml} / \mathrm{g}] \times 10 \times \mathrm{Hb}[\mathrm{g} / \mathrm{dl}] \times \mathrm{Fs}_{\mathrm{a}} \mathrm{O}_{2}+0,03[\mathrm{ml} / \mathrm{l} / \mathrm{mmHg}]$ $\times \mathrm{p}_{\mathrm{a}} \mathrm{O}_{2}[\mathrm{mmHg}]$

In der Literatur wurde gezeigt, dass das aktuelle Rauchverhalten Einfluss auf die Messwerte für $\mathrm{s}_{\mathrm{a}} \mathrm{O}_{2}$ und den $\mathrm{Hb}$ haben kann $[7,8]$. Ziel dieser Untersuchung ist die Überprüfung der vorhandenen Normwerte für den arteriellen Sauerstoffgehalt unter Berücksichtigung des aktuellen Rauchverhaltens, basierend auf einer populationsbasierten Stichprobe klinisch gesunder Erwachsener.

\section{Patienten und Methodik}

Im Rahmen einer populationsbasierten Querschnittsstudie (Study of Health in Pomerania-SHIP-1) [9] wurden zwischen März 2003 Juli 20063300 Freiwillige zwischen 25 und 85 Jahren untersucht. Im Rahmen der Untersuchung erfolgten unter anderem ein Computergestütztes Interview, eine ärztliche Untersuchung inkl. EKG und Echokardiografie.

Von den 3300 Probanden erklärten sich 1708 bereit, an einer Lungenfunktionsuntersuchung und Spiroergometrie teilzuneh- men. Eine ausführliche Charakterisierung der Studienpopulation und des Studiensamples findet sich bei Koch et al. [10].

Alle Teilnehmer gaben ihr schriftliches Einverständnis. Die Studie entsprach den Prinzipien der Deklaration von Helsinki entsprechend dem positiven Votum der lokalen Ethikkommission.

Im Rahmen der Lungenfunktion/Spiroergometrie wurden Blutgasanalysen aus arterialisiertem Kapillarblut sowie der Hämoglobinwert bestimmt. Hierzu wurde ein Ohrläppchen mit Nonivamid/Nicoboxil (Finalgon Creme ${ }^{\circledR}$, Boehringer Ingelheim) mindestens 10 Minuten vor der Blutentnahme eingerieben, um einen freien Kapillarblutfluss, unabhängig von lokalen Manipulationen, zu erreichen. Mittels einer sterilen Nadel wurde das Ohrläppchen inzidiert, und nur Proben mit freiem, unmanipuliertem Blutfluss wurden verwendet. Das Blut wurde in $55 \mu$ Kapillaren (Clinitubes $^{\circledR}$, Radiometer, Kopenhagen, Dänemark) gesammelt und direkt in den Blutgasanalysator eingegeben (Verzögerung zwischen Entnahme und Analyse <30 Sekunden). Blutgase und Hämoglobinwert wurden in einem ABL 510 (Radiometer, Kopenhagen, Dänemark) bestimmt. Das Gerät wurde täglich entsprechend der Herstellervorgaben kalibriert.

Im Rahmen des computergestützten Interviews wurde das Rauchverhalten der Probanden erfragt. Als Raucher wurden Probanden gezählt, die angaben, täglich zu rauchen.

Alle Untersuchungen wurden von trainierten und zertifizierten Mitarbeitern durchgeführt. Es erfolgten jährliche Kontrollen durch ein externes Data Safety and Monitoring Committee.

\section{Ausschlusskriterien}

Für die vorliegende Untersuchung wurden Probanden mit einer manifesten Anämie $(\mathrm{Hb}<7 \mathrm{mmol} / \mathrm{l})$ sowie einer Hypoxämie $\left(\mathrm{s}_{\mathrm{a}} \mathrm{O}_{2}\right.$ $<93 \%$ ) ausgeschlossen.

\section{Statistische Auswertung}

Der arterielle Sauerstoffgehalt wurde nach o.g. Formel bestimmt. Um Referenzwertgleichungen für den Sauerstoffgehalt zu bestimmen, wurden Gleichungen für die Vorhersage der 5., 10., 50. und 90. Perzentile mittels quantiler Regression, adjustiert für Alter und Geschlecht und Raucherstatus (aktuell Raucher: ja/nein) ermittelt. Als untere Normwertgrenze wurde die 5. Perzentile angesehen. Die Analysen erfolgten mit SPSS Statistics Version 22 (SPSS Inc., IBM München, Deutschland) und Stata 13.1 (Stata Corporation, College Station, TX, USA).

\section{Ergebnisse \\ $\nabla$}

Von 1080 Probanden lagen alle Daten vor. 62 Probanden wurden aufgrund einer Anämie oder einer Hypoxämie ausgeschlossen.

Somit standen Daten von 1018 Probanden (496 Männer) für die Analysen zur Verfügung. Die Probanden waren zwischen 26 und 80 Jahre alt (Median 50 Jahre, Interquartilsabstand 40 - 61 Jahre). 223 Probanden waren aktuelle Raucher. Deskriptive Statistiken finden sich in $\bullet$ Tab. 1.

Die Verteilung der Messwerte für $\mathrm{Hb}, \mathrm{s}_{\mathrm{a}} \mathrm{O}_{2}$ und $\mathrm{p}_{\mathrm{a}} \mathrm{O}_{2}$ sowie den berechneten arteriellen Sauerstoffgehalt sind in $\bullet$ Abb. 1 wiedergegeben.

Die für Alter, Geschlecht und Rauchstatus korrigierten Normwertgleichungen sind in $\bullet$ Tab. 2 angegeben. Die grafischen Darstellungen finden sich in $\bullet$ Abb. 2 und $\bullet$ Abb. 3 .

Der über die Normwertformel prognostizierte Median und der untere Normwert für den arteriellen Sauerstoffgehalt nehmen bei Männern mit zunehmendem Alter kontinuierlich ab. Raucher 
Tab.1 Beschreibung der Studienpopulation.

\begin{tabular}{|c|c|c|c|c|c|c|}
\hline Alter [Jahre] & männlich & Raucher & $\mathrm{s}_{\mathrm{a}} \mathrm{O}_{2}[\%]$ & $\mathrm{Pa}_{2} \mathrm{O}_{2}[\mathrm{mmHg}]$ & $\mathrm{Hb}[\mathrm{mmol} / \mathrm{l}]$ & $\mathrm{c}_{\mathrm{a}} \mathrm{O}_{2}[\mathrm{ml} / \mathrm{l}]$ \\
\hline \multirow[t]{2}{*}{$26-40$} & 126 & 85 & 97 & 87,4 & 8,8 & 193 \\
\hline & $47,6 \%$ & $32,1 \%$ & $96,2-97,6$ & $78,2-93,0$ & $8,1-9,4$ & $180-207$ \\
\hline \multirow[t]{2}{*}{$41-60$} & 224 & 119 & 96,6 & 81,4 & 8,9 & 193 \\
\hline & $46,1 \%$ & $24,5 \%$ & $95,7-97,3$ & $74,8-87,8$ & $8,3-9,4$ & $182-205$ \\
\hline \multirow[t]{2}{*}{$61-80$} & 146 & 19 & 96,2 & 77,4 & 8,7 & 190 \\
\hline & $54,7 \%$ & $7,1 \%$ & $95,1-96,9$ & $71,6-83,5$ & $8,2-9,2$ & $179-201$ \\
\hline
\end{tabular}

Qualitative Daten: Anzahl, Prozent

Quantitative Daten: Median, Interquartilsabstand
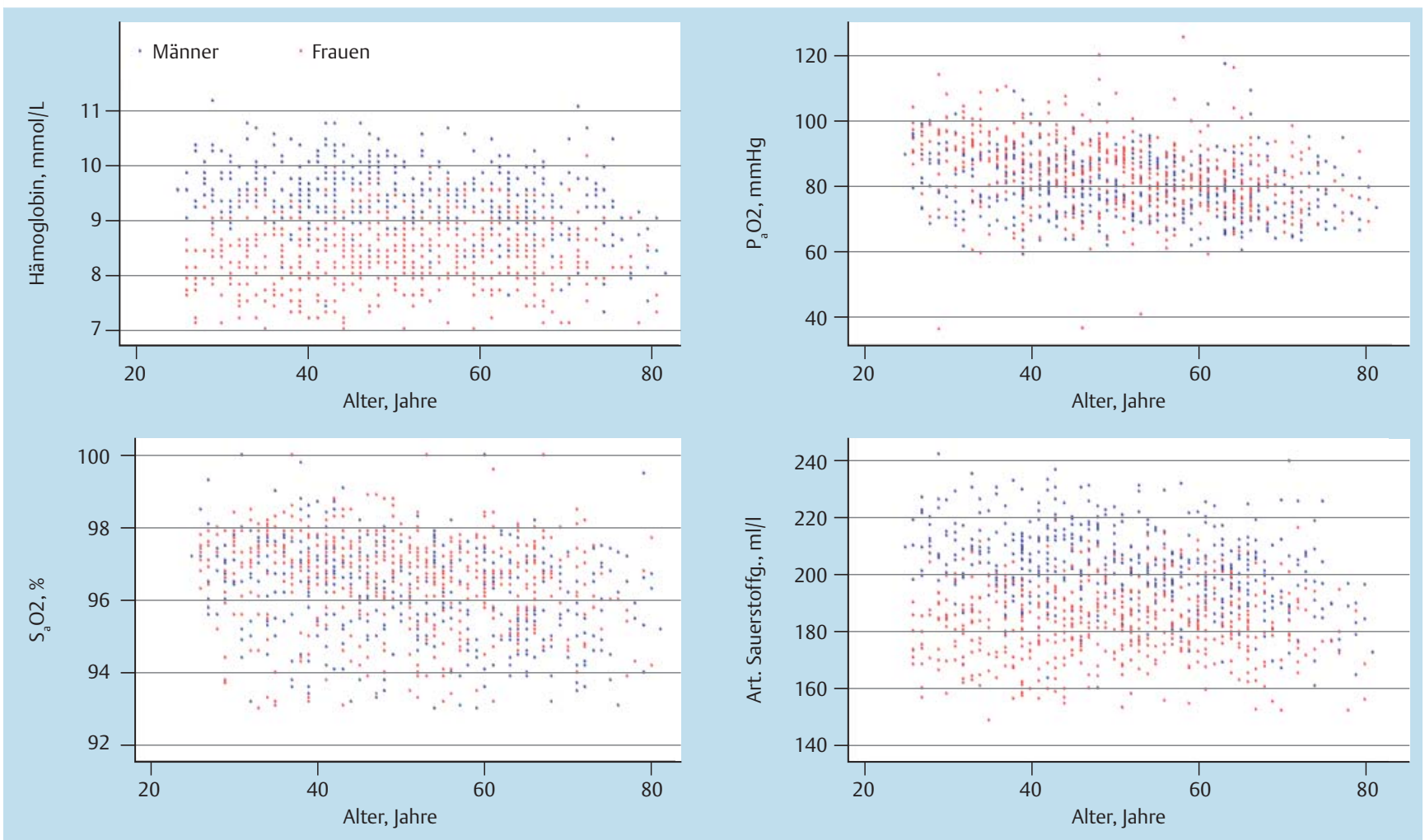

Abb. 1 Alters- und geschlechtsabhängige Verteilung von $\mathrm{SaO}_{2}, \mathrm{P}_{\mathrm{a}} \mathrm{O}_{2}$, Hämoglobin und Sauerstoffgehalt, gemessen im arterialisierten Kapillarblut.

und Nichtraucher unterscheiden sich nicht signifikant ( $\mathrm{p}$ für die 90., 50., 10. und 5. Perzentile: 0,39; 0,86; 0,74; 0,85).

Bei Frauen ist der prognostizierte Median weitgehend altersunabhängig, liegt aber für Raucherinnen mit ca. $5 \mathrm{ml} / \mathrm{l}$ signifikant höher als für Nichtraucherinnen $(\mathrm{p}<0,005)$. Die prognostizierte 90., 10. und 5. Perzentile und damit auch der untere Normwert steigen bei Frauen bis zum 55. Lebensjahr zunächst kontinuierlich an, um danach deutlich abzufallen. Für die 90. Perzentile liegt der prognostizierte Wert für Raucherinnen signifikant über dem für Nichtraucherinnen $(p<0,001)$. Für die 10. und 5. Perzentile ist der Unterschied nicht signifikant ( $p=0,21$ bzw. $p=0,61)$.

\section{Diskussion}

Im Gegensatz zu den bisher publizierten Normwerten für $\mathrm{c}_{\mathrm{a}} \mathrm{O}_{2}$ liegt nun erstmals eine alters- und geschlechtsbezogene Normwertformel unter Beachtung des Raucherstatus vor.

Die in dieser Arbeit berechneten Werte für den arteriellen Sauerstoffgehalt (Median $191 \mathrm{ml} / \mathrm{l} \mathrm{O}_{2}$ ) sind mit den 1984 von Mertzlufft und Zander veröffentlichten, direkt gemessenen Werten, vergleichbar. Diese gaben bei Männern einen Mittelwert von 203,3 ml/1 (Raucher 203,6 ml/1, Nichtraucher 203,9 ml/1) an. Für Frauen lag der gemessene Mittelwert bei 185,8 ml/1 (Raucherinnen $185,4 \mathrm{ml} / \mathrm{l}$, Nichtraucherinnen $186,0 \mathrm{ml} / \mathrm{l}$ ) [3]. In beiden Arbeiten zeigt sich ein höherer Sauerstoffgehalt bei Männern mit einem deutlichen Abfall im Alter. Der altersbedingte Abfall bei den Frauen ist geringer ausgeprägt, vermutlich durch den mit der Menopause ansteigenden Hämoglobingehalt ( $\bullet$ Abb.1).

Im Vergleich zu den von Siggaard-Andersen publizierten Normwerten [4] liegt die Stärke der aktuellen Untersuchung in der größeren Fallzahl (1018 vs. 35) und genaueren Charakterisierung der Studienpopulation.

Die vorgeschlagenen Normwertformeln berücksichtigen das aktuelle Rauchverhalten der Probanden. Allerdings war der Unterschied im unteren Normwert zwischen Rauchern und Nichtrauchern sowohl bei Männern als auch bei Frauen statistisch nicht signifikant.

Der populationsbasierte Ansatz der vorliegenden Untersuchung erlaubt die Erstellung von alters- und geschlechtsspezifischen Normwertgleichungen. Die Methode der quantilen Regression 


\begin{tabular}{|c|c|}
\hline Perzentile & $\mathrm{C}_{\mathrm{a}} \mathrm{O}_{2}$ \\
\hline \multicolumn{2}{|l|}{ Männer } \\
\hline 5. & $205.3287-0.4753^{*}$ Alter $^{1}+0.6451 *$ Rauchen $^{2}$ \\
\hline 10. & $209.9698-0.4551 *$ Alter $+0.9148 *$ Rauchen \\
\hline 50. & $220.6915-0.3622 *$ Alter $+0.3006 *$ Rauchen \\
\hline 90. & $238.3345-0.3874 *$ Alter $+2.5574 *$ Rauchen \\
\hline \multicolumn{2}{|l|}{ Frauen } \\
\hline 5. & $154.2522+0.3929 *(\text { Alter } / 10)^{3}-0.1934 * \ln (\text { Alter } / 10)^{*}(\text { Alter } / 10)^{3}-1.6104 *$ Rauchen \\
\hline 10. & $155.7249+0.4336^{*}(\text { Alter } / 10)^{3}-0.2083^{*} \ln (\text { Alter } / 10)^{*}(\text { Alter } / 10)^{3}+3.3117^{*}$ Rauchen \\
\hline 50. & $178.6177+0.0659 *$ Alter $+5.1642 *$ Rauchen \\
\hline 90. & $184.5065+0.5745^{*}(\text { Alter } / 10)^{3}-0.2756^{*} \operatorname{In}(\text { Alter } / 10)^{*}(\text { Alter } / 10)^{3}+8.0262 *$ Rauchen \\
\hline
\end{tabular}

Tab.2 Normwertformeln für den arteriellen Sauerstoffgehalt $\mathrm{c}_{\mathrm{a}} \mathrm{O}_{2}$.

${ }^{1}$ Alter in Jahren

${ }^{2} 0$ aktuell Nichtraucher, 1 aktuell Raucher

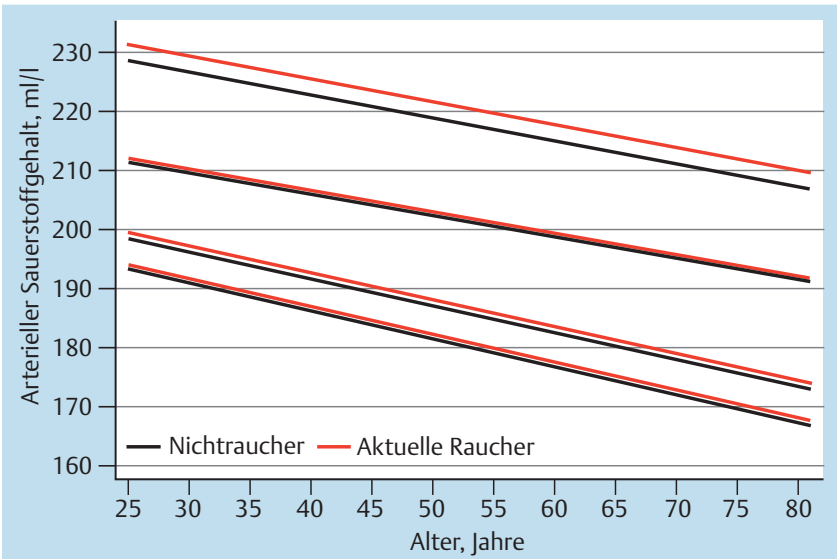

Abb.2 Sauerstoffgehalt $\mathrm{caO}_{2}$ im arterialisierten Kapillarblut bei Männern. Angegeben sind der Median sowie die 5., 10. und 90. Perzentile, jeweils getrennt für Raucher und Nichtraucher. Die 5. Perzentile entspricht dem unteren Normwert.

ist besonders robust gegenüber Ausreißern und macht keine besonderen Annahmen über die Verteilung der Messwerte.

Die vorgelegte Untersuchung stellt erstmals an einer norddeutschen Population erhobene Normwerte vor.

Ungeachtet des mathematischen Zusammenhangs der drei untersuchten Größen $\left(\mathrm{Hb}, \mathrm{p}_{\mathrm{a}} \mathrm{O}_{2}, \mathrm{~s}_{\mathrm{a}} \mathrm{O}_{2}\right)$ kann aus klinischer Sicht keine vollständige gegenseitige Kompensation erfolgen. Zwar entwickelt sich bekanntermaßen bei chronisch hypoxischen Erkrankungen eine Polyglobulie, Grenzen sind hier jedoch z.B. durch die Blutviskosität gesetzt. Auch das Herzminutenvolumen als wesentlicher Faktor für das Sauerstoffangebot konnte in dieser Untersuchung nicht berücksichtigt werden.

\section{Limitationen}

Für die Erstellung der Normwerte wurde für die Sauerstoffbindungskonstante des Hämoglobins der Wert von 1,39 ml/g angenommen. Auch wenn der Wert mit 1,39 ml/g der theoretisch maximalen Sauerstoffaufnahme des Hämoglobins entspricht, liegt der tatsächliche Wert der Sauerstoffbindungskapazität wohl eher zwischen $1,31 \mathrm{ml} / \mathrm{g}$ und $1,34 \mathrm{ml} / \mathrm{g}[5,11]$. Letztlich ist es jedoch lediglich wichtig, bei Erstellung und Anwendung der Normwertgleichung den gleichen Faktor zu verwenden.

Eine weitere Limitation liegt in der Verwendung von arterialisiertem Kapillarblut zur Bestimmung der Sauerstoffsättigung sowie des Sauerstoffpartialdruckes. Eine Bestimmung der Blutgase aus arteriellem Blut erschien im Rahmen einer populationsbasierten Untersuchung jedoch unverhältnismäßig.

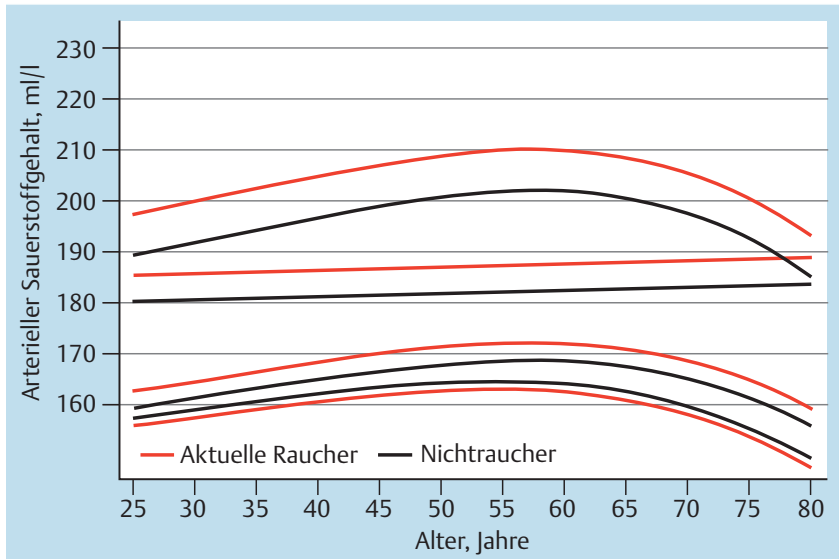

Abb.3 Sauerstoffgehalt $\mathrm{caO}_{2}$ im arterialisierten Kapillarblut bei Frauen. Angegeben sind der Median sowie die 5., 10. und 90. Perzentile, jeweils getrennt für Raucher und Nichtraucher. Die 5. Perzentile entspricht dem unteren Normwert.

Der Wert dieser Normwerte liegt aus unserer Sicht darin, eine klinische Orientierung zu geben und dem Anwender zu vergegenwärtigen, dass weder Sauerstoffsättigung noch Hämoglobingehalt für sich betrachtet eine suffiziente Aussage über die Sauerstoffversorgung zulassen.

\section{Hinweis \\ $\nabla$}

Die SHIP-Studie ist Teil des Forschungsverbundes Community Medicine des Universitätsklinikums Greifswald. Dieses ist gefördert durch das Bundesministerium für Bildung und Forschung (BMBF) (01ZZ9603, 01ZZ0103, 01ZZ0403, 01ZZ0701) und das Sozialministerium des Landes Mecklenburg-Vorpommern. Außerdem ist diese Arbeit Teil des Forschungsprojektes Greifswald Approach to Individualized Medicine (GANI_MED). Das GANI_MED Konsortium wird durch das BMBF und durch das Kultusministerium Mecklenburg-Vorpommern (03IS2061A) gefördert. Die Studiengruppe wird weiterhin durch das Kompetenznetz Asthma/COPD, gefördert durch das BMBF (FKZ 01GI08810888), unterstützt.

\section{Interessenkonflikt \\ $\nabla$}

Die Autoren geben an, dass kein Interessenkonflikt besteht. 


\section{Literatur}

1 Köhler D, Haidl P. Sauerstoff in der Medizin. Pneumologie 2011; 65: $25-35$

2 Magnussen H, Kirsten A-M, Köhler D et al. Leitlinien zur Langzeit-Sauerstofftherapie - Deutsche Gesellschaft für Pneumologie und Beatmungsmedizin e.V. Pneumologie 2008; 62: 748-756

3 Mertzlufft $F$, Zander $R$. Normal values of oxygen concentration in human blood. Adv Exp Med Biol 1984; 180: 581-590

4 Siggaard-Andersen 0 , Wimberley PD, Fogh-Andersen $N$ et al. Arterial oxygen status determined with routine $\mathrm{pH} /$ blood gas equipment and multi-wavelength hemoximetry: reference values, precision, and accuracy. Scand J Clin Lab Invest Suppl 1990; 203: 57-66

5 Hüfner G. Ueber die Quantität Sauerstoff, welche 1 Gramm Hämoglobin zu binden vermag. Z Physiol Chem 1878; 6: 386-394

6 Burnett RW, Covington AK, Fogh-Andersen $N$ et al. International Federation of Clinical Chemistry (IFCC). Scientific Division. Committee on pH, Blood Gases and Electrolytes. Approved IFCC recommendations on whole blood sampling, transport and storage for simultaneous determination of pH, blood gases and electrolytes. Eur J Clin Chem Clin Biochem 1995; 33: 247-253

7 Goldbourt U, Medalie JH. Characteristics of smokers, non-smokers and ex-smokers among 10,000 adult males in Israel. II. Physiologic, biochemical and genetic characteristics. Am J Epidemiol 1977; 105: 75-86

8 Tirlapur VG, Gicheru K, Charalambous BM et al. Packed cell volume, haemoglobin, and oxygen saturation changes in healthy smokers and non-smokers. Thorax 1983; 38: 785 - 787

9 Völzke H, Alte D, Schmidt CO et al. Cohort Profile: The Study of Health in Pomerania. Int J Epidemiol 2011; 40: 294-307

10 Koch B, Schäper C, Ittermann $T$ et al. Reference values for cardiopulmonary exercise testing in healthy volunteers: the SHIP study. European Respiratory Journal 2008; 33: 389-397

11 Lumb AB. Oxygen. In: Nunn's Applied Respiratory Physiology. 7th ed. Edinburgh: Churchill Livingston Elsevier; 2010: 179-216 